Supporting Information for

\title{
Comprehensive Lipidomics Analysis Reveals the Effects of Different Omega-3 Polyunsaturated Fatty Acid-Rich Diets on Egg Yolk Lipids
}

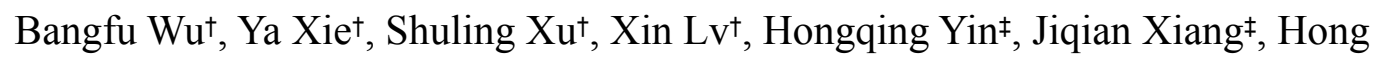
Chen ${ }^{\dagger}$, Fang Wei ${ }^{\dagger}, *$

tKey Laboratory of Oilseeds Processing of Ministry of Agriculture, Key Laboratory of Biology and Genetic Improvement of Oil Crops of Ministry of Agriculture, P. R. China and Hubei Key Laboratory of Lipid Chemistry and Nutrition, Oil Crops Research Institute of the Chinese Academy of Agricultural Sciences, P. R. China

‡Enshi Autonomous Prefecture Academy of Agricultural Sciences, Enshi, Hubei 445002, P.R. China

${ }^{*}$ Corresponding author: Fang Wei

Address: Xudong 2nd Road, Wuhan, Hubei, 430062, P. R. China.

Tel: +86-27-86711669

Fax: +86-27-86822291

E-mail: willasa@163.com 
The Supporting Information includes following items:

Page 4-5

Figure S1. Some examples of lipid species identification via MS/MS spectra matching in MS-DIAL with an integrated LipidBlast database.

Figure S2. Separation of TAG (68:11) $942.7534\left(\left[\mathrm{M}+\mathrm{NH}_{4}\right]^{+}\right)$: (A) extracted

Page 6 ion chromatogram (EIC) of TAG (68:11) 942.7534, (B) isomer 1 (eluted at $7.99 \mathrm{~min})$ tentatively identified as (16:0-20:5-22:6) by MS/MS, and (C) isomer 2 (eluted at $8.15 \mathrm{~min}$ ) tentatively identified as TAG (18:2-18:3-22:6).

Page 7

Figure S3. LC retention map of lipid classes identified under the positive (upper panel) and negative (lower panel) scan modes. Y-axis indicated the average $\mathrm{m} / \mathrm{z}$ of each lipid species, and X-axis indicated the average RT (retention time, min) of each lipid species. Each dot represented an individual lipid species and dots with the same color represented a lipid class.

Figure S4. Dynamic changes and variation tendencies of AA-lipids in egg yolk samples from hens fed with different n-3 PUFA-rich diets during dietary supplementation (n=3): (A) flaxseed group (F); (B) Schizochytrium sp. residue

Page 8 group (SR) and (C) flaxseed + Schizochytrium sp. residue group (F+SR). The contents of AA-lipid in different forms in egg yolk samples from hens after different dietary treatments for 5 weeks $(n=3)$. The concentrations were calculated based on the weight of dry egg yolk for lipid extraction. Different letters indicate statistically significant differences $(p<0.05)$.

Figure S5. Analysis of significantly differential lipid species in F vs Control, SR vs Control and F+SR vs Control. OPLS-DA score plots (A) and volcano plot analysis (B) of F vs Control, SR vs Control and F+SR vs Control. A number of

Page 9 significantly differential lipid species were screened out by using the criteria of $p$ $<0.05$ and $\mathrm{FC}>2$ (up-regulated) or $\mathrm{FC}<0.5$ (down-regulated) in the volcano plot. Significantly differential lipid species were shown as a red (up) or green (down) dot, whereas a black dot represented no significant difference of lipid species.

Figure S6. Heatmap visualization of dynamic changes of significantly differential lipid species in F vs control (A), SR vs control (B) and F+SR vs control (C). Colors Page $10 \quad$ represent the contents of different lipid classes with red indicating a high content of lipids and blue indicating a low content. Data shown in each cell is mean value of contents of three replicates.

Figure S7. Pathway analysis showing changing lipid metabolism after dietary supplementation with different n-3 PUFA sources for lying hens: (A) flaxseed (F);

(B) Schizochytrium sp. residue (SR) and (C) flaxseed + Schizochytrium sp. residue $(\mathrm{F}+\mathrm{SR})$. The $\mathrm{X}$-axis represents the pathway impact, and the Y-axis represents the -log ( $p$-value). The size and color of the circle represent the pathway impact and pathway significance, respectively. 
Excel sheet

Supplementary table sheet 1
Table S1. Lipid Contents and Fatty Acid Compositions (\% by Weight of Total Fatty Acids) of Schizochytrium sp. Residue and Flaxseed.

\section{Excel sheet}

Supplementary

table sheet 2
Table S2. The Weekly Averages of Actual Feed Intake for Hens in Each Group (g/hen/day).

Excel sheet

Supplementary

Table S3. Lipid Species Identified and Quantified in Egg Yolk Samples from table sheet 3 Hens Fed with Different Diets ( $n=3, \mu \mathrm{g} / \mathrm{g}$ dry egg yolk).

Excel sheet

Supplementary

Table S4. Molecular Species and Contents of ALA-Lipids in Different Groups table sheet 3 after 5 Weeks of Dietary Supplementation ( $n=3, \mu \mathrm{g} / \mathrm{g}$ dry egg yolk).

\section{Excel sheet}

Supplementary

Table S5. Molecular Species and Contents of EPA-Lipids in Different Groups table sheet 4 after 5 Weeks of Dietary Supplementation ( $n=3, \mu \mathrm{g} / \mathrm{g}$ dry egg yolk).

\begin{tabular}{ll}
\hline $\begin{array}{l}\text { Excel sheet } \\
\begin{array}{l}\text { Supplementary } \\
\text { table sheet } 5\end{array}\end{array}$ & $\begin{array}{l}\text { Table S6. Molecular Species and Contents of DPA-Lipids in Different Groups } \\
\text { after } 5 \text { Weeks of Dietary Supplementation ( } n=3, \mu \mathrm{g} / \mathrm{g} \text { dry egg yolk). }\end{array}$ \\
\hline $\begin{array}{l}\text { Excel sheet } \\
\text { Supplementary } \\
\text { table sheet } 6\end{array}$ & $\begin{array}{l}\text { Table S7. Molecular Species and Contents of AA-Lipids in Different Groups } \\
\text { after } 5 \text { Weeks of Dietary Supplementation ( } n=3, \mu \mathrm{g} / \mathrm{g} \text { dry egg yolk). }\end{array}$ \\
\hline $\begin{array}{l}\text { Excel sheet } \\
\text { Supplementary } \\
\text { table sheet } 7\end{array}$ & $\begin{array}{l}\text { Table S8. The List of Most Prominently Differential Lipid Species in Egg Yolk } \\
\text { after Treatments with Different n-3 PUFA-rich Diets for } 5 \text { Weeks, Selected }\end{array}$ \\
\hline
\end{tabular}



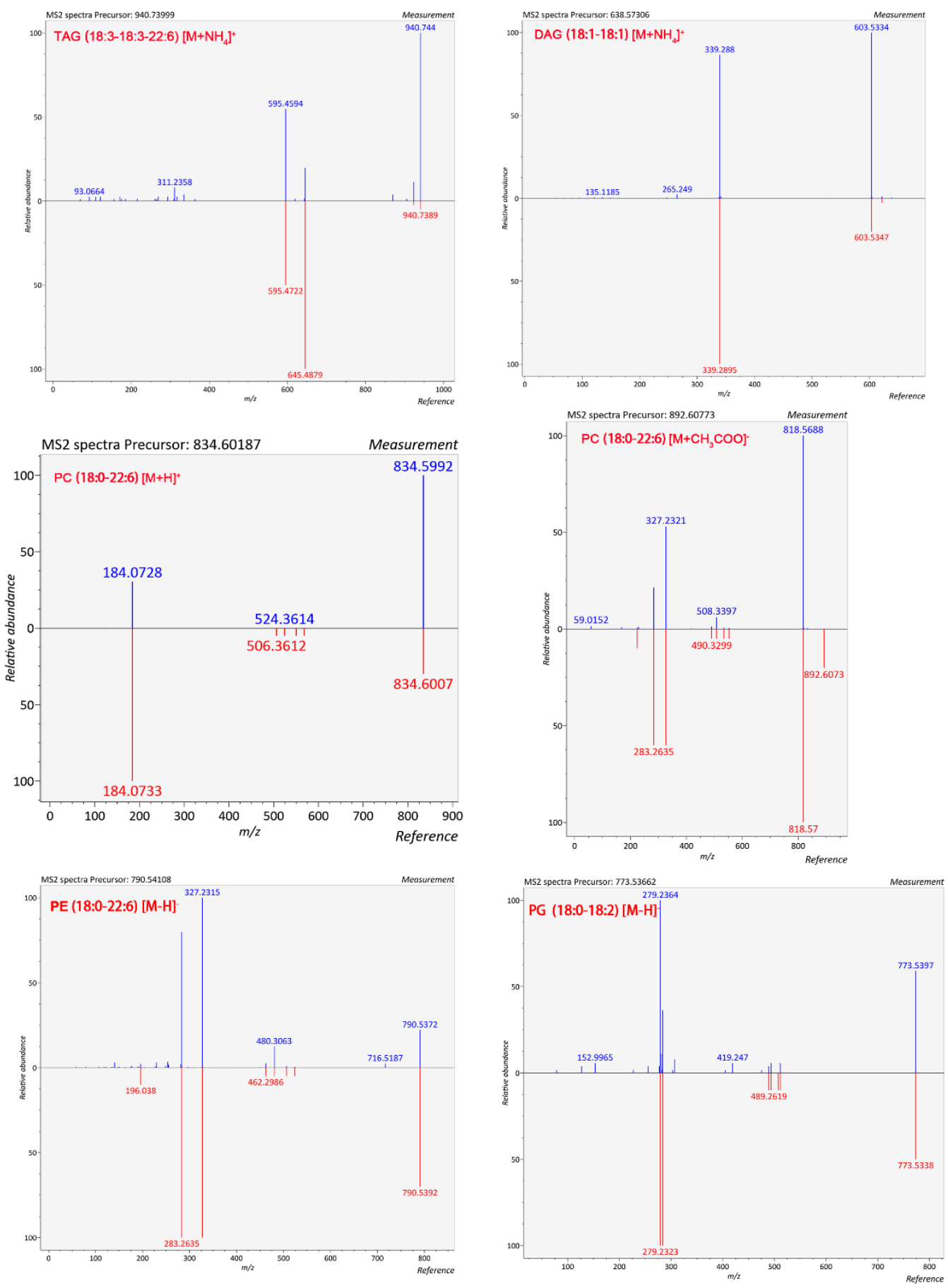

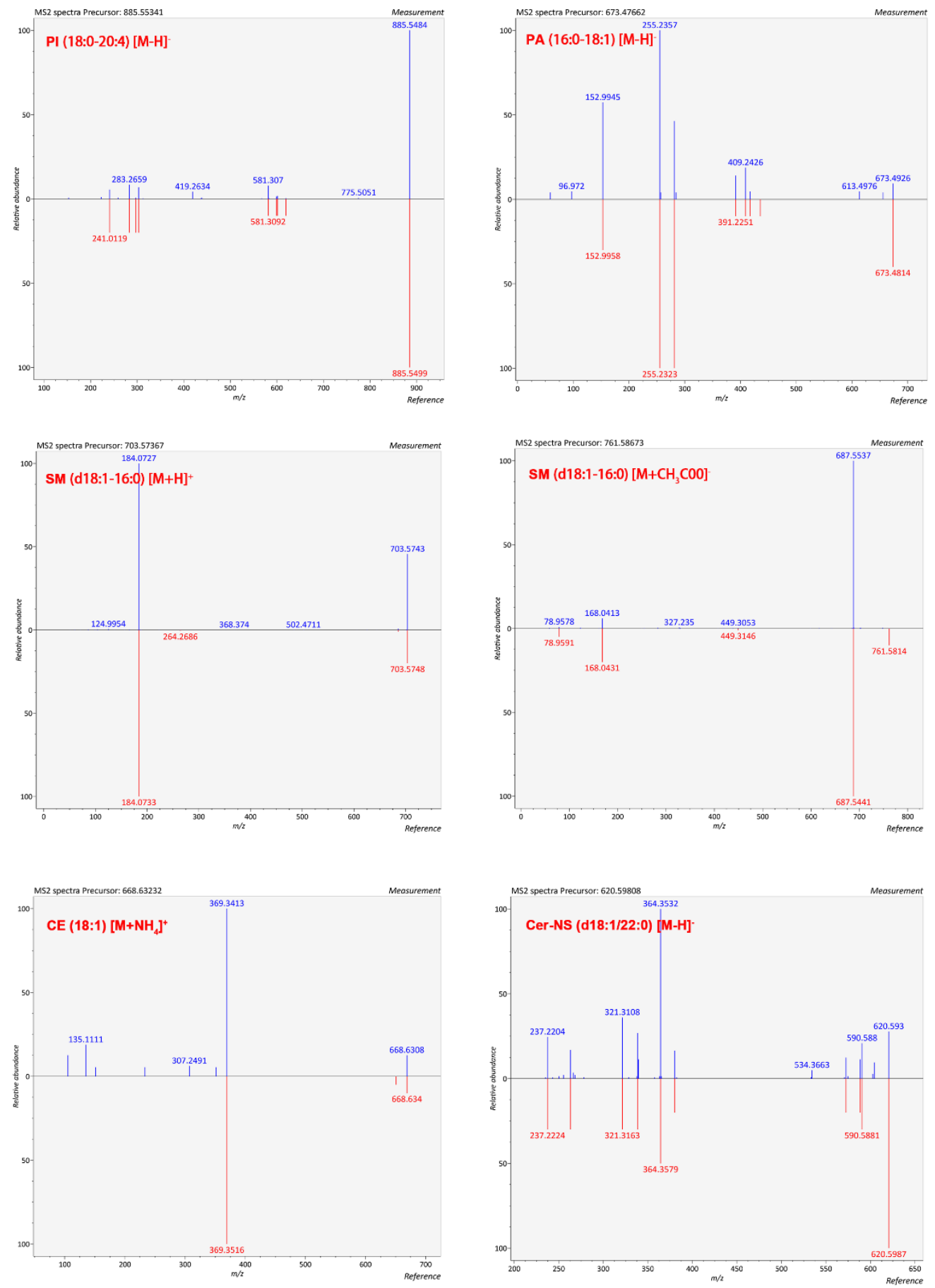

Figure S1. Some examples of lipid species identification via MS/MS spectra matching in MS-DIAL with an integrated LipidBlast database. 


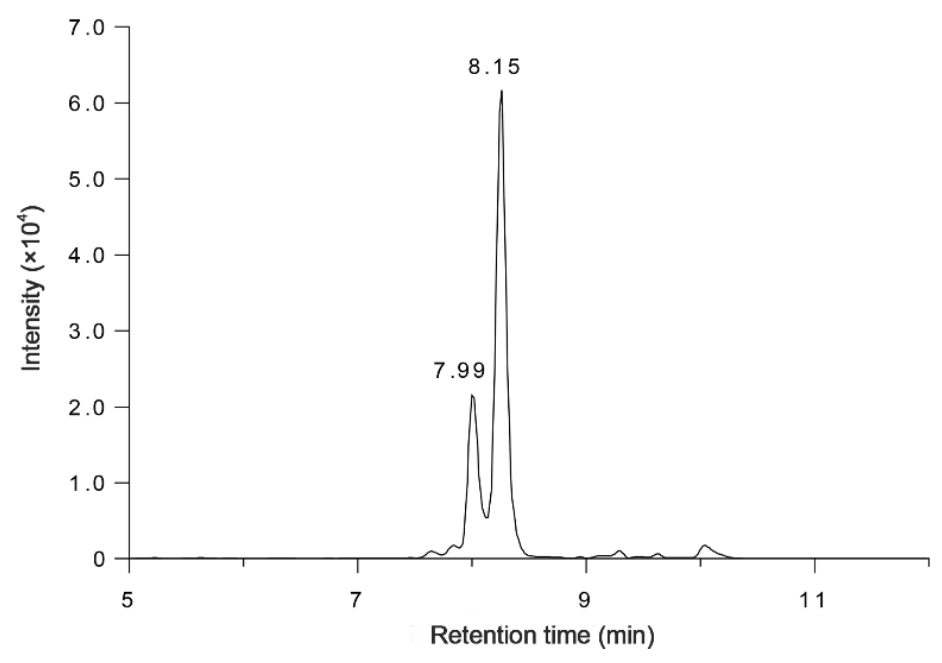

(A)
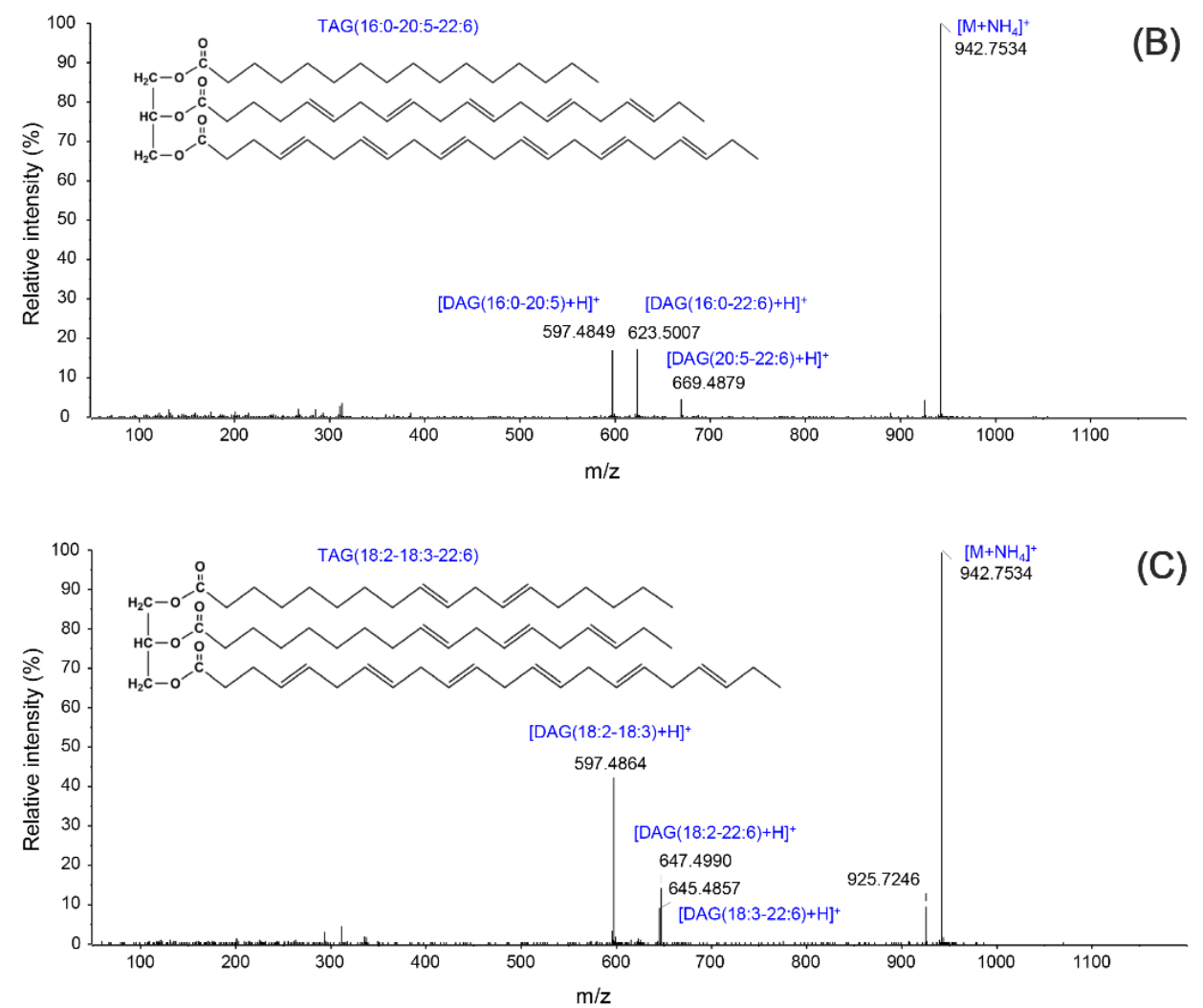

Figure S2. Separation of TAG (68:11) $942.7534\left([\mathrm{M}+\mathrm{NH} 4]^{+}\right)$: (A) extracted ion chromatogram (EIC) of TAG (68:11) 942.7534, (B) isomer 1 (eluted at $7.99 \mathrm{~min}$ ) tentatively identified as (16:0-20:5-22:6) by MS/MS, and (C) isomer 2 (eluted at $8.15 \mathrm{~min}$ ) tentatively identified as TAG (18:2-18:3-22:6). 

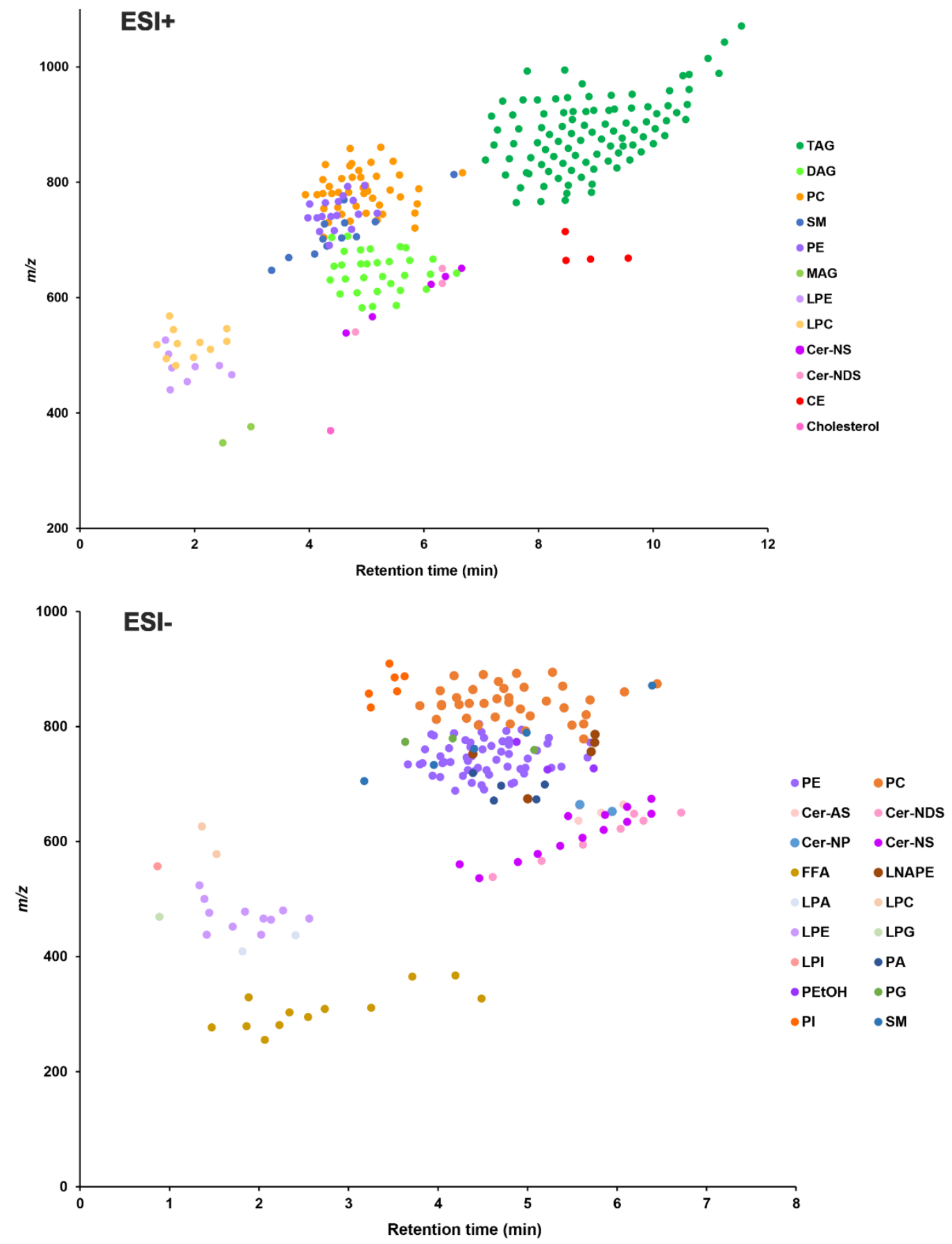

Figure S3. LC retention map of lipid classes identified under the positive (upper panel) and negative (lower panel) scan modes. Y-axis indicated the average $\mathrm{m} / \mathrm{z}$ of each lipid species, and $\mathrm{X}$-axis indicated the average RT (retention time, min) of each lipid species. Each dot represented an individual lipid species and dots with the same color represented a lipid class. 

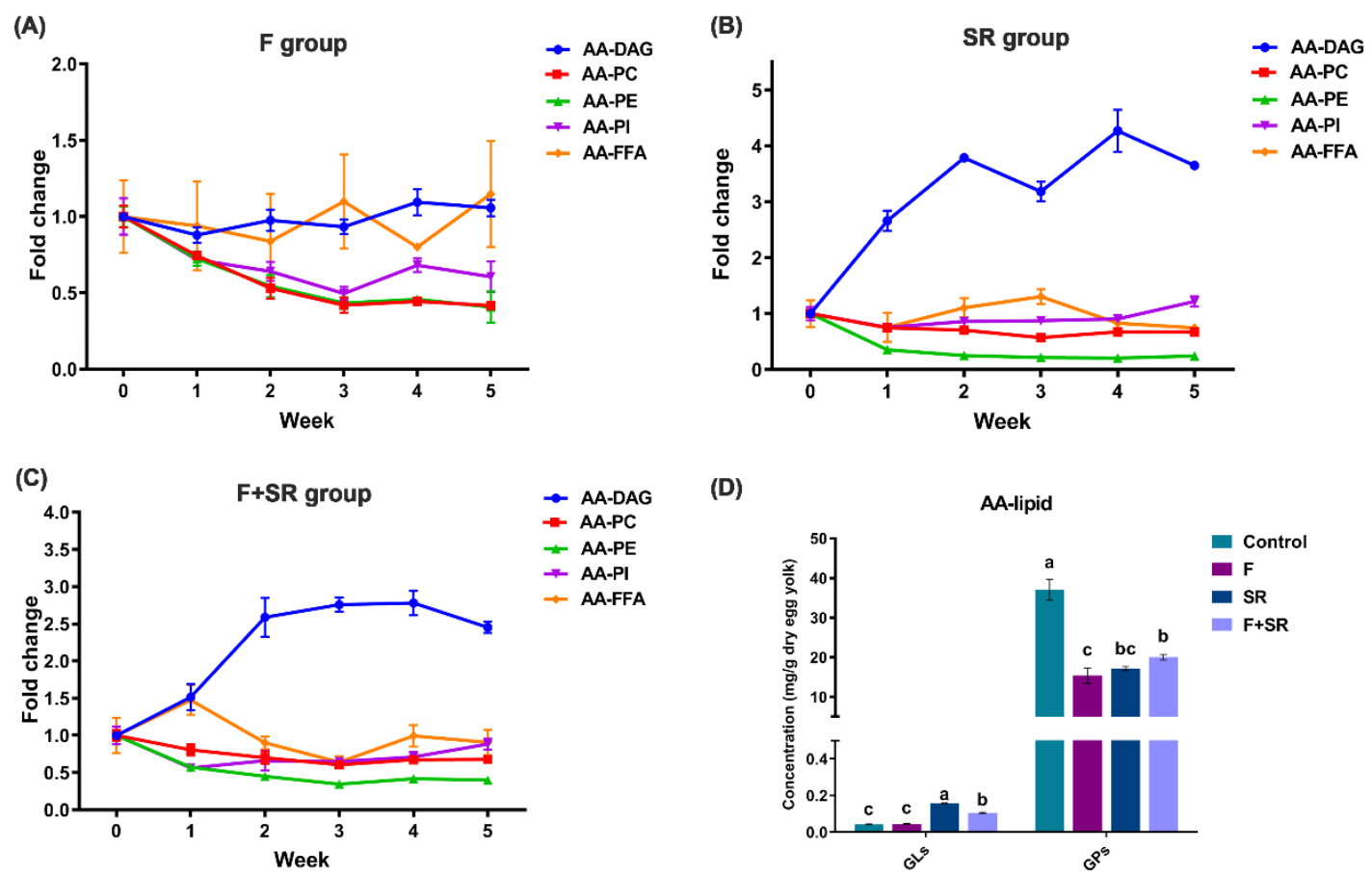

Figure S4. Dynamic changes and variation tendencies of AA-lipids in egg yolk samples from hens fed with different n-3 PUFA-rich diets during dietary supplementation (n=3): (A) flaxseed group (F); (B) Schizochytrium sp. residue group (SR) and (C) flaxseed + Schizochytrium sp. residue group (F+SR). The contents of AA-lipids in different forms in egg yolk samples from hens after different dietary treatments for 5 weeks $(n=3)$. The concentrations were calculated based on the weight of dry egg yolk for lipid extraction. Different letters indicate statistically significant differences $(\mathrm{p}<0.05)$. 
(A)

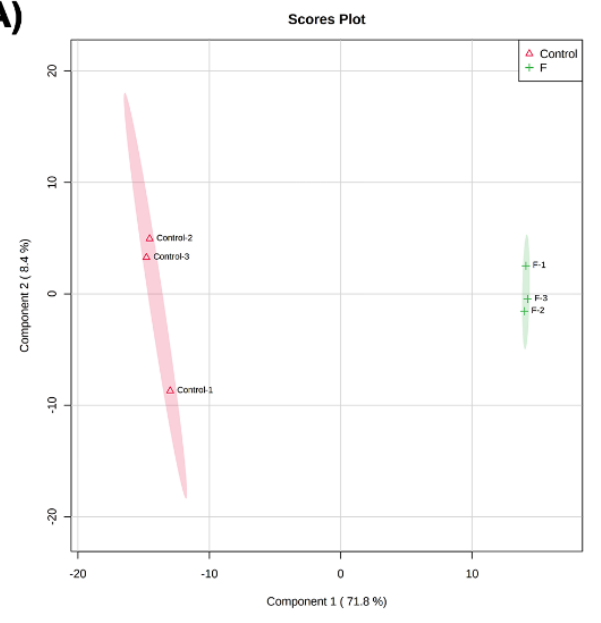

(B)

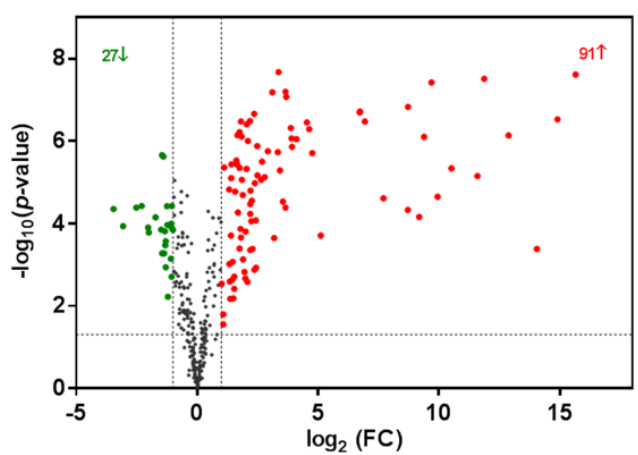

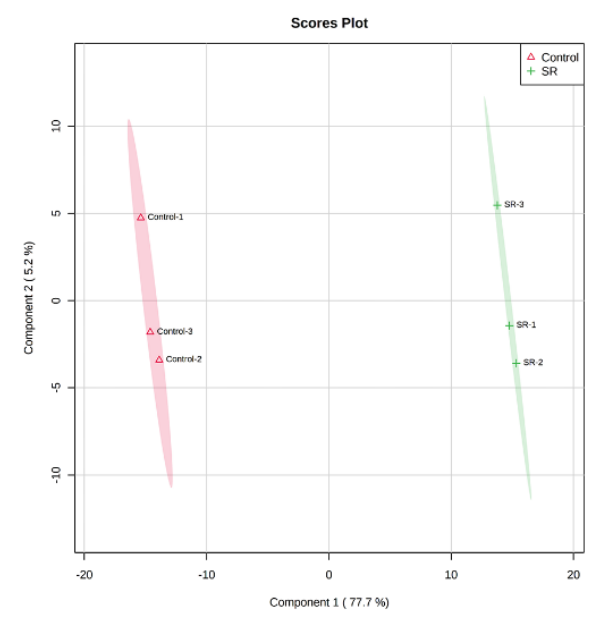

SR vs Control

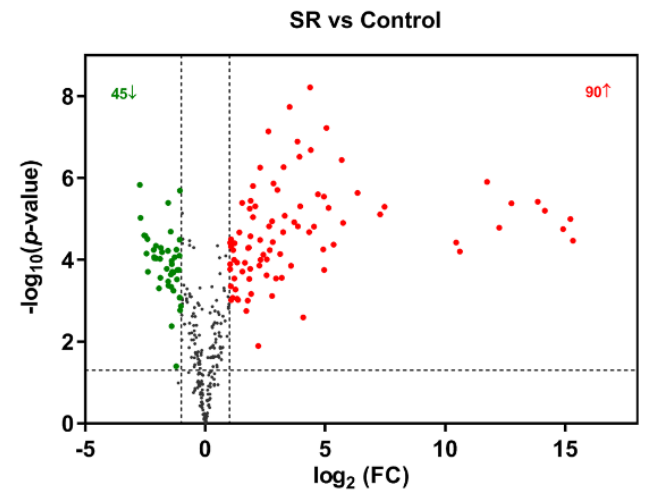

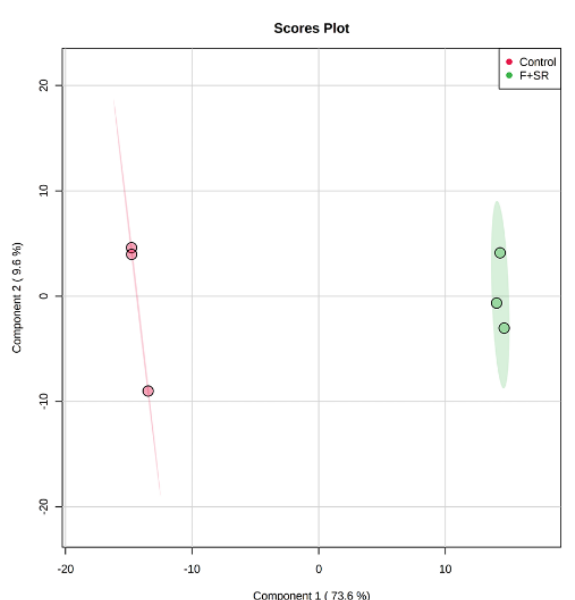

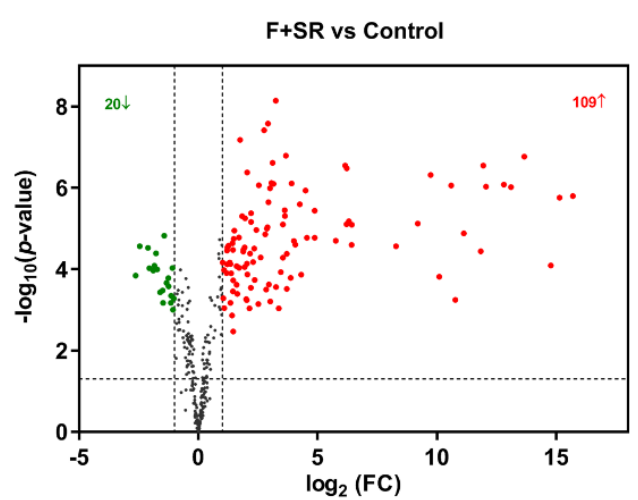

Figure S5. Analysis of significantly differential lipid species in F vs Control, SR vs Control and F+SR vs Control. OPLS-DA score plots (A) and volcano plot analysis (B) of F vs Control, SR vs Control and F+SR vs Control. A number of significantly differential lipid species were screened out by using the criteria of $p<0.05$ and FC $>2$ (upregulated) or FC $<0.5$ (down-regulated) in the volcano plot. Significantly differential lipid species were shown as a red (up) or green (down) dot, whereas a black dot represented no significant difference of lipid species. 
(A) F vs Control

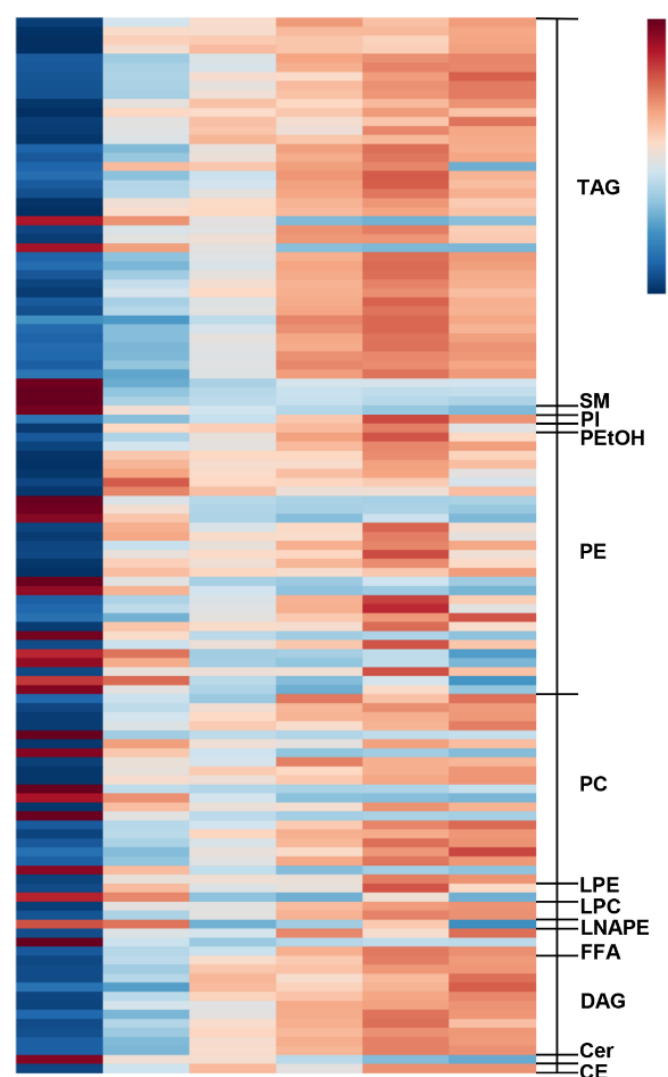

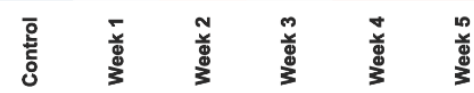

(B) SR vs Control

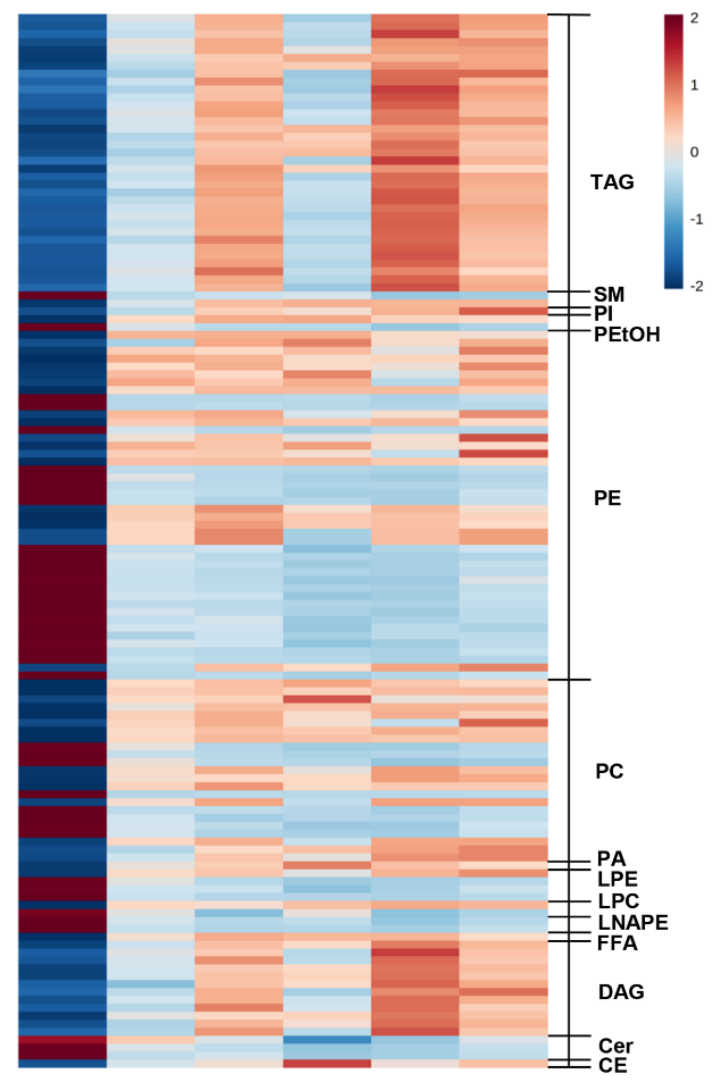

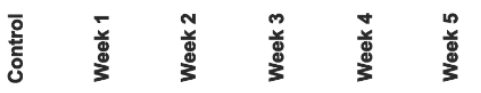

(C) F+SR vs Control

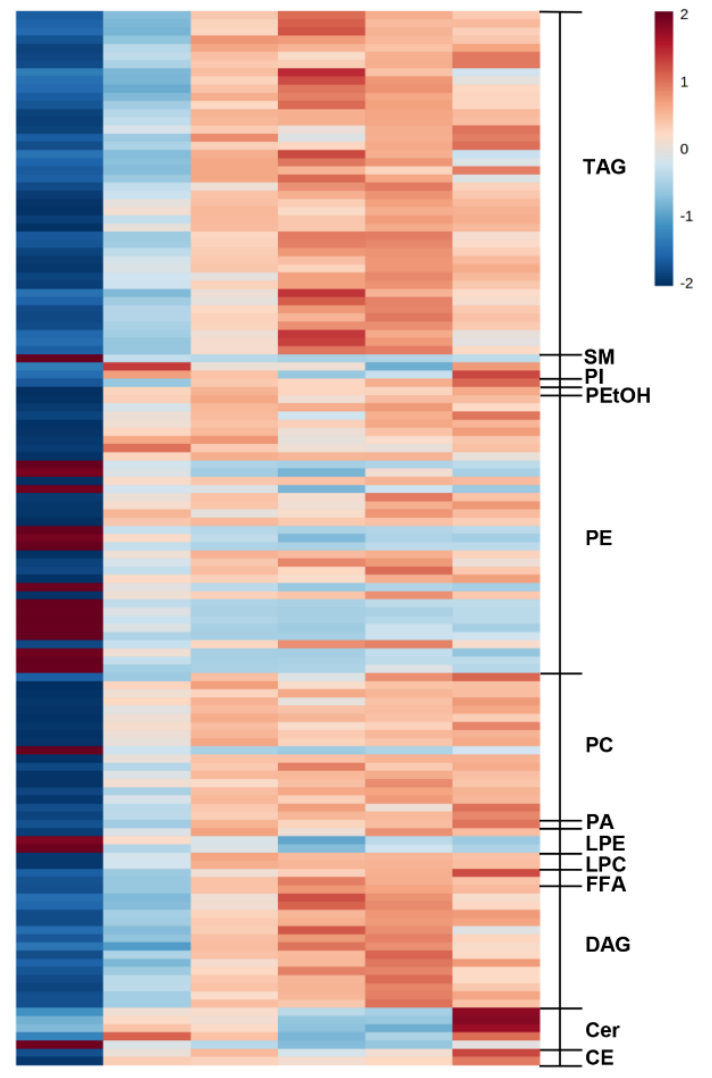

竞

Figure S6. Heatmap visualization of dynamic changes of significantly differential lipid species in F vs Control (A), SR vs Control (B) and F+SR vs Control (C). Colors represent the contents of different lipid classes with red indicating a high content of lipids and blue indicating a low content. Data shown in each cell is mean value of contents of three replicates. 
(A)



(B)

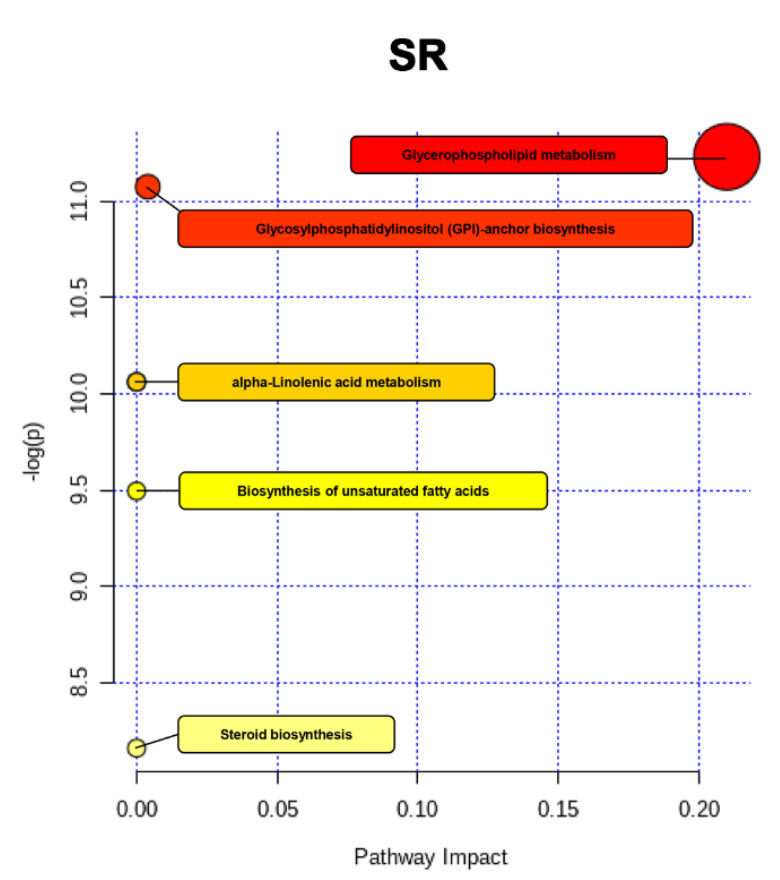

(C)

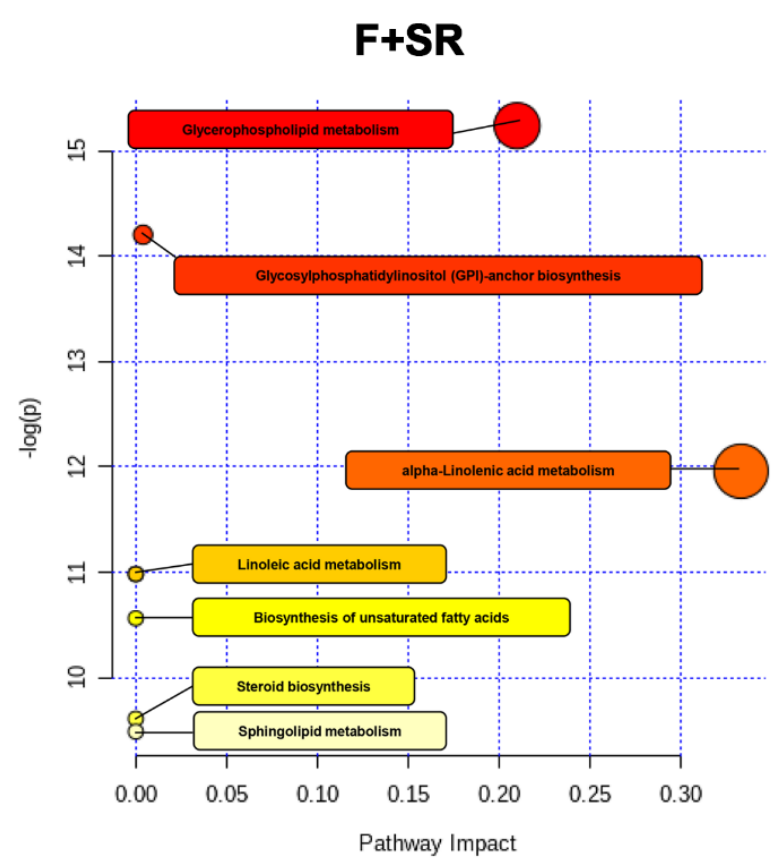

Figure S7. Pathway analysis showing changing lipid metabolism after dietary supplementation with different n-3 PUFA sources for lying hens: (A) flaxseed (F); (B) Schizochytrium sp. residue (SR) and (C) flaxseed + Schizochytrium sp. residue (F+SR). The X-axis represents the pathway impact, and the Y-axis represents the -log ( $p$-value). The size and color of the circle represent the pathway impact and pathway significance, respectively. 